\title{
The impact of the molecular classification of glioblastoma on the interpretation of therapeutic clinical trial results
}

\author{
Lauren S. Singer ${ }^{1}$, Alexander Z. Feldman², Robin A. Buerki ${ }^{3}$, Craig M. Horbinski ${ }^{2,4,5}$, Rimas V. Lukas ${ }^{5,6}$, \\ Roger Stupp ${ }^{4,5,6,7}$
}

${ }^{1}$ Department of Neurology, Rush University, Chicago, IL, USA; ${ }^{2}$ Department of Pathology, Northwestern University, Chicago, IL, USA; ${ }^{3}$ Department of Neurology, Case-Western University, Chicago, IL, USA; ${ }^{4}$ Department of Neurological Surgery, Lurie Comprehensive Cancer Center, Chicago, IL, USA; ${ }^{5}$ Malnati Brain Tumor Institute and Lurie Comprehensive Cancer Center, Chicago, IL, USA; ${ }^{6}$ Department of Neurology, Lurie Comprehensive Cancer Center, Chicago, IL, USA; ${ }^{7}$ Department of Medicine (Section of Hematology \& Oncology), Lurie Comprehensive Cancer Center, Chicago, IL, USA

Contributions: (I) Conception and design: RV Lukas, R Stupp; (II) Administrative support: RV Lukas, R Stupp; (III) Provision of study materials or patients: All authors; (IV) Collection and assembly of data: LS Singer, AZ Feldman; (V) Data analysis and interpretation: All authors; (VI) Manuscript writing: All authors; (VII) Final approval of manuscript: All authors.

Correspondence to: Roger Stupp, MD. Northwestern Medicine, 676 N. St Clair, Suite 2210, Chicago, IL 60611, USA.

Email: Roger.stupp@northwestern.edu.

\begin{abstract}
In 2016, the World Health Organization (WHO) released the most recent update to the classification of central nervous system tumors. This update has led to the reshaping of tumor identification and subsequently changed current understanding of treatment options for patients. Moreover, the restructuring of the classification of central nervous system tumors to include molecular markers has led to the need to re-evaluate how to interpret pivotal trials. These trials originally enrolled patients purely based upon histologic diagnoses without the use of adjunctive, and frequently diagnostic molecular testing. With this new paradigm also comes the need to assess how one should incorporate molecular markers into current trials as well as shape future trials. First, we will discuss updates on the molecular classification of glioblastoma (GBM) (and its histologic mimics). This will be followed by a review of key pivotal trials which have defined our standard of care for glioblastoma within the context of molecular classification of their study populations. This will be followed by preliminary results of ongoing phase 3 cooperative group trials for high-grade gliomas that were initiated prior to routine molecular classification of tumors and how one could interpret these results in light of advances in molecular classification. Finally, we will end with suggestions for future clinical trial design with a focus on enrollment based upon molecular diagnostics.
\end{abstract}

Keywords: Astrocytoma; clinical trial; glioblastoma (GBM); IDH; neuropathology

Submitted Mar 17, 2021. Accepted for publication Apr 01, 2021.

doi: $10.21037 /$ cco-21-33

View this article at: http://dx.doi.org/10.21037/cco-21-33

\section{Introduction}

Great progress has been made in understanding the molecular biology and pathogenesis which drives infiltrating gliomas. Consequently, tumor nosology has evolved from a purely morphological to a more refined and dynamic classification incorporating histology, immunohistochemistry, and most recently, molecular testing. Using histology alone, many central nervous system tumors may show similar features, though are now known to have different molecular underpinnings, which often influences prognosis and treatment options. Integration of molecular studies has led to more refined diagnoses and allows further clarification of the pathophysiologic processes that contribute to gliomagenesis, proliferation, tumor spread, and, ultimately, survival. This review will focus 
on grade IV infiltrating astrocytic tumors, predominantly glioblastomas (GBM), and the impact advanced molecular testing has on diagnosis and treatment.

The most recent update of the World Health Organization (WHO) classification of central nervous system tumors stems from 2016, with a new update on the horizon for 2021, one that will most certainly incorporate even more molecular-based classifications than its predecessor (1). As all pivotal trials which have defined our standard of care were conducted prior to the current era of molecular classification, we will discuss how this impacts our interpretation of the results of these trials (2). As ongoing, large, cooperative group trials were initiated prior to the widespread incorporation of molecular diagnostics into the classification of high-grade glioma classification, how best to interpret their results will also be discussed. Finally, commentary will be made on how best to design future clinical trials with respect to the rapid advances in molecular diagnostics and its impact on tumor classification.

\section{Updates on the molecular classification of GBM and its histologic mimics}

Most studies establishing the current standard of care for GBM utilized the third and fourth editions of the WHO Classification of Tumours of the Central Nervous System (published in 2000 and 2007, respectively), which relied exclusively on histology to establish a diagnosis $(3,4)$. At that time, criteria for the diagnosis of GBM was histologically determined and consisted of an infiltrating glioma with astrocytic features, mitotic activity and microvascular proliferation and/or necrosis. However, we now know that a purely histologic approach combines neoplasms that have distinct molecular underpinnings, differing prognoses, and, increasingly, different therapeutic options. Official steps toward an integrated molecular and histologic classification began with the fifth edition of the WHO Classification of Tumours of the Central Nervous System (published in 2016) and subsequent updates of the Consortium to Inform Molecular and Practical Approaches to CNS Tumor Taxonomy-Not Official WHO (cIMPACT-NOW) (5-9). The upcoming WHO edition, expected to be published in 2021 or 2022, will represent yet another extension of this molecular-histologic integration.

Because of these modifications, the diagnostic criteria for a WHO grade 4 GBM have been refined to include an IDH-wildtype (wt), H3-wildtype diffusely infiltrating glioma with microvascular proliferation, necrosis and/or one or more of the following molecular alterations: TERT promoter mutation, EGFR amplification, and/or combined whole chromosome gain of 7 with loss of chromosome 10 $(7,9)$. Tumors meeting only these molecular criteria have similarly poor outcomes, whether or not they also meet histologic criteria for GBM $(9,10)$. The term "glioblastoma" is now reserved only for these IDH-wildtype astrocytomas with histologic or molecular features of GBM. Array-based methylation profiling has uncovered six different subclasses of IDH-wildtype GBMs [receptor tyrosine kinase (RTK) I/II/III, MYCN, mesenchymal and midline] which show differing age profiles, molecular alterations, and expression profiles $(11,12)$. Notably, what were once considered distinct entities, gliosarcomas and giant cell GBMs, do not represent separate methylation subclasses, but instead classify as part of the IDH-wildtype GBM methylation class family $(11,12)$.

Astrocytoma, IDH-mutant, WHO grade 4, is defined as a diffusely infiltrating astrocytic glioma with an IDH1 or IDH2 mutation in conjunction with microvascular proliferation, necrosis and/or CDKN2A/B homozygous deletions $(8,9)$. These tumors frequently also harbor mutations in ATRX and TP53 and are less aggressive than IDH-wildtype GBMs $(13,14)$. Functional loss of ATRX results in alternative lengthening of telomeres, a process that appears mutually exclusive with TERT promoter mutations, another method of telomere maintenance. The latter is mostly seen in IDH-wildtype GBMs as well as IDH-mutant, 1p/19q-codeleted oligodendrogliomas (15).

Oligodendrogliomas are defined as diffuse gliomas with an IDH1 or IDH2 mutation and whole-arm $1 \mathrm{p} / 19 \mathrm{q}$-codeletion (5). Such tumors usually possess TERT promoter mutations as a method of telomere maintenance, this in contrast to the ATRX mutations of IDH-mutant astrocytomas. WHO grade 3 anaplastic oligodendrogliomas are defined as having these molecular features plus 6 or more mitoses per 10 high powered fields, microvascular proliferation, and/or necrosis. Anaplastic oligodendrogliomas sometimes lack the classic rounded, relatively uniform, hyperchromatic nuclei with perinuclear halos, and they may show more marked nuclear atypia, sometimes even with a prominent astrocytic appearance (5). In the pre-molecular era, such findings might have been confused with a GBM, especially the small cell variant (16). The distinction, however, is important because, as a group, patients with anaplastic oligodendrogliomas still have much longer median survival than IDH-wildtype GBMs, or even IDH-mutant grade 3 or grade 4 astrocytomas (16). 
Though histone H3-mutant gliomas are sometimes thought of as pediatric tumors, in reality, the age range is considerably more extensive, with more adult cases being detected with better screening (17-19). As the name implies, the diffuse midline glioma, H3 K27Mmutant, WHO grade 4, shows a predilection for midline locations-pons, thalamus and spinal cord being most common $(6,18)$. Outcomes in adults with this tumor are poor and appear on par with that of IDH-wildtype GBM (18). Diffuse glioma, H3.3 G34-mutant, WHO grade 4 , is a newly described entity most commonly seen in the cerebral hemispheres of adolescents and young adults, though this tumor has been reported in older patients $(9,19)$. Some histone-driven tumors show histological features similar to that of a conventional GBM, whereas others are deceptively bland. Subsets (especially of G34-mutant gliomas) can mimic embryonal neoplasm $(9,19)$. Notably, H3.3 G34-mutant gliomas are frequently negative for Olig2 (a glial lineage marker), with concomitant loss of ATRX staining and strong p53 staining (the latter two corresponding to molecular alterations in ATRX and TP53, respectively) (19). The median survival of patients with this tumor appears to be between that of an IDH-wildtype GBM and a WHO grade 4 IDH-mutant astrocytoma (20).

Array-based methylation profiling of histologicallydefined cerebellar GBMs has shown that subsets of these tumors represent a unique methylation class with the current recommended nomenclature of "high grade astrocytoma with piloid features" $(9,21,22)$. The microscopic appearance of this tumor is usually high grade, though with variable histology, with not all cases showing piloid features (21). This entity has a unique molecular profile: IDH-wildtype and H3-wildtype, with MAPK pathway gene alterations (e.g., NF1, BRAF, FGFR1, etc.), and frequent alterations in ATRX and CDKN2A/B (22). The outcome in these tumors is more favorable than that of IDH-wildtype GBMs, though unfavorable when compared to conventional pilocytic astrocytomas (22).

In many cases, the distinction between anaplastic pleomorphic xanthoastrocytomas (PXAs) and GBMs (particularly those with an epithelioid phenotype) on purely histologic grounds is impossible without the aid of more advanced molecular testing. BRAF p.V600E mutations and concomitant homozygous CDKN2A/B deletions are seen in approximately $70 \%$ of grade 2-3 PXAs (12). However, this combination of alterations can also be seen in IDH-wildtype
GBMs, albeit rarely (23). At the current time, there is very limited experience in integrating and targeting druggable mutations in the first-line treatment of GBM. However, in the recurrent setting an increasing number of case reports suggest that in select patients targeting BRAF or NTRK will lead to meaningful responses (24). Array-based methylation profiling is able to stratify most of these challenging tumors into the more prognostically favorable PXA versus the less favorable GBM methylation subclasses (23).

\section{Impact of molecular classification of GBM on interpretation of pivotal trials}

Prior to the 2016 WHO classification and c-IMPACT NOW publications, therapeutic trials demonstrated the importance of molecular neuropathology in GBM. In 2005, the phase 3 EORTC/NCIC trial demonstrated the value of early systemic chemotherapy in the treatment of histologically defined GBMs (25). The median survival for patients who received radiotherapy (RT) + temozolomide (TMZ) was 14.6 (95\% CI, 12.3-16.8) as compared to 12.1 months (95\% CI, 11.2-13.0) for patients who received RT alone. Median progression free survival was 5.0 (95\% CI, 4.2-5.5) vs. 6.9 (95\% CI, 5.8-8.2) months for patients receiving RT and RT+TMZ, respectively. In this study, 573 patients were enrolled across 85 centers with roughly equal numbers being distributed to RT versus RT plus TMZ. Of these patients, slides were available for central review in $85 \%$ with the histologic diagnosis of GBM being confirmed in $93 \%(n=185)$ with $3 \%(n=16)$ being anaplastic astrocytoma or anaplastic oligoastrocytoma. A remaining $1 \%(\mathrm{n}=6)$ did not have sufficient tissue for a definitive diagnosis. This trial, demonstrating the efficacy of RT plus concomitant and adjuvant temozolomide (TMZ), was conducted prior to the routine inclusion of molecular characteristics in the classification of infiltrating gliomas. However, the molecular marker of O6-methylguanineDNA-methyltransferase (MGMT) promoter methylation was able to establish a clear relationship between promoter methylation and benefit from TMZ (26). This molecular marker is not incorporated in the current WHO classification system, although its clinical relevance is substantial.

Shortly after this trial, in 2009, a study of the mutational status of gliomas revealed the presence of mutations in IDH1 and IDH2 in a subset of these tumors (14). Of grade 4 tumors analyzed in this study, 6 of 123 primary GBMs and 11 of 13 secondary GBMs (those which arose from lower grade tumors) demonstrated these IDH mutations. IDH 
mutations were also more strongly associated with mutations in TP53 (80\%) while IDH-wt tumors were more strongly associated with alterations of PTEN, EGFR, CDKN2A/ $\mathrm{B}(74 \%)$. Individuals with IDH mutations were found to be younger (mean age of 32 for GBM). Overall, patients with an IDH-mutant tumor showed improved median overall survival of 31 months compared to 15 months for IDHwildtype GBM (P=0.002).

Thus, we can presume that the patient population of EORTC/NCIC were predominantly IDH-wt given the composition of the trial population of newly diagnosed GBM with a median age of 56 years. Moreover, IDH-wt GBM are estimated to make up $90 \%$ of all GBM while IDH-mutated tumors represent $8-10 \%$ of all GBM (27). As such, the EORTC/NCIC can be viewed as representative of a viable treatment in newly diagnosed GBMs. While probable, it is not completely certain that these results extend to IDH-mutated astrocytomas. The positive trial results of $T M Z$ in recurrent anaplastic astrocytoma, a significant subset of which were likely IDH-mutated, would lend support to this idea (28). Despite the molecular advances following this trial, this treatment regimen ultimately holds validity for at least IDH-wildtype GBM even in the age of new molecular diagnostic criteria.

The phase $3 \mathrm{EF}-14$ trial evaluated tumor-treating fields (TTFields) in newly diagnosed GBM (29). Improved survival, progression free survival, and landmark survival were observed in patients who received TTFields in addition to TMZ. In this study, retrospective pathology review was performed evaluating $1 \mathrm{p} / 19 \mathrm{q}$ co-deletion, EGFR amplification, and IDH1 mutation status. IDH mutational status was determined by immunohistochemistry for IDH1$\mathrm{R} 132 \mathrm{H}$, which accounts of approximately $90 \%$ of IDH mutations in diffusely infiltrating astrocytomas (27). In this trial, 695 patients were included with 379 samples tested for IDH1 R132H (29). Of the samples available for testing, $93 \%(n=353)$ of patients were demonstrated to be IDHwt GBM. An additional 6.5\% (n=25) were IDH1 R132Hmutated grade 4 astrocytomas. The mutational status of EGFR and the presence/absence of $1 \mathrm{p} 19 \mathrm{q}$ co-deletion were also evaluated by fluorescent in situ hybridization. A total of 364 samples were tested for EGFR amplification and 371 for $1 \mathrm{p} 19 \mathrm{q}$ codeletion. Of those studied, $39.8 \%$ $(n=145)$ showed amplification of EGFR representing GBMs. Only $0.3 \%(n=2)$ showed co-deletion of $1 p 19 q$ and may be grouped in the IDH1 R132H mutant diffusely infiltrating high grade gliomas. Overall, this trial population is predominantly composed of IDH-wildtype GBM with a minority population of approximately $6.5 \%$ representing IDH1 R132H mutant diffuse gliomas.

The primary end point for this study was median progression free survival which was 6.7 months for patients who received TTFields and 4.0 months in patients who did not (29). The secondary endpoint of overall survival also demonstrated improvement in the investigational arm with 20.9 months and 16.0 months in patients receiving TTFields in addition to $\mathrm{TMZ}$ and $\mathrm{TMZ}$ alone, respectively ( $\mathrm{HR} 0.63$; 95\% CI, 0.52-0.76, $\mathrm{P}<0.001$ ). In this study improvement in relevant clinical endpoints was seen in both IDH-wt GBM and grade 4 IDH-mutant astrocytomas. This lends support that this therapeutic approach may be beneficial in more than one type of highgrade infiltrating glioma, particularly high grade IDHmutant astrocytomas.

\section{Impact of molecular classification of GBM on interpretation of key ongoing trials for high- grade gliomas}

In building upon the results of these pivotal trials, there are currently two long term ongoing trials which we will discuss. The results of these, will need to be considered within the context of the contemporary molecular neuropathology landscape. Both trials were developed prior to the new classification system, but preliminary results have led to the understanding and modification of these studies based on molecular markers. The phase III CATNON study (NCT00626990 and EORTC 26053-22054) focuses on newly diagnosed non-1p/19q co-deleted grade 3 anaplastic gliomas (30). In these trials, individuals were randomized either to RT alone, RT with concurrent TMZ, RT with adjuvant TMZ up to 12 cycles, or RT with concurrent and adjuvant TMZ to 12 cycles in a $2 \times 2$ matrix design. Initial analysis of the entire study population, presented at ASCO 2019, showed no significant benefit with concurrent TMZ with respect to 5 -year survival at $50.2 \%$ with concurrent TMZ and $52.7 \%$ without concurrent TMZ. It did, however, show overall survival for those receiving adjuvant TMZ was significantly improved compared to those who did not receive it (55.9\% as compared to $44.1 \%$; HR 0.65 ). Notably however, a post hoc analysis of the IDH-mutant subset of CATNON patients proved the value of TMZ. Median overall survival was 19 months in IDH-wt tumors (which would be equivalent to GBM IDHwt) as compared to 116 months in IDH-mutant tumors, demonstrating the difference in natural history of these two tumor types (31). 
In this interim analysis IDH mutated tumors benefited from adjuvant TMZ (IDH mutated HR 0.41, IDH-wt HR 1.05) as well as with concurrent TMZ (IDH mutated HR 0.67, IDH-wt HR 1.27, $\mathrm{P}=0.06$ ). These findings reflect the benefit of alkylating chemotherapy, specifically TMZ, in IDH-mutant infiltrating astrocytomas. MGMT promoter methylation is more frequently present in IDH-mutant glioma than in IDH-wt GBM and is well known as being prognostically beneficial and predictive of TMZ response in GBM. Interim analysis of CATNON revealed high percentage of MGMT methylation (70\%) but did not demonstrate significant benefit for either concurrent $(\mathrm{P}=0.09)$ or adjuvant $(\mathrm{P}=0.16)$ TMZ. This may be secondary to the study being underpowered to detect such a difference. Similar findings regarding lack of benefit for MGMT methylated IDH-mutant tumors have been seen in other retrospective series of IDH-mutant lower-grade glioma (32). Again, statistical power may play a role in this. These findings may also be influenced by the development of TMZ-induced hypermutation $(33,34)$. It is worth noting that CATNON was not powered to analyze effects based on molecular subtypes as molecular markers were not fully understood at the time this study was designed. Thus, there is some uncertainty on how to optimally interpret null results.

Another multicenter international phase III study, CODEL (NCT00878146; EORTC 26081/NRG 1071/ Alliance N0577), is investigating the effect of RT and chemotherapy on newly diagnosed, high-risk chromosome $1 \mathrm{p} / 19 \mathrm{q}$ co-deleted oligodendrogliomas (35). The use of a molecular marker for CODEL eligibility facilitates a more homogenous population than CATNON, which includes both IDH-wt and IDH mutated infiltrating astrocytic tumors. At its inception, CODEL randomized anaplastic oligodendroglioma subjects into 3 treatment arms: RT alone, TMZ alone, and RT with concurrent and adjuvant TMZ. Preliminary data showed that progression free survival was significantly shorter in TMZ-alone than in patients who also received RT (HR 3.12, 95\% CI, 1.26-7.69, $\mathrm{P}=0.014)$. This led to early closure of the TMZ monotherapy arm. Subsequently, following the publication of the RTOG 9402 results CODEL was further restructured to two arms: RT followed by 6 cycles of procarbazine, CCNU, and vincristine (PCV) versus RT with concurrent and 12 cycles adjuvant TMZ. In addition to anaplastic WHO grade 3 tumors, WHO grade 2 oligodendroglioma with "high-risk" features (any of: age $\geq 40$, age $<40$ and subtotal resection/biopsy, radiographic progression, intractable seizures) are also eligible.

Additional molecular subtype analysis has been performed for IDH status in 35/36 (97\%) patients by immunohistochemistry for IDH-R132H (35). Of these, $30 / 35$ (86\%) were IDH-mutant. Of the 5 deemed IDHwt, 2 were confirmed as such by sequencing and the other 3 were considered IDH-wt by the immunohistochemistry results alone. When analysis was performed accounting for IDH mutation status, progression free survival remained shorter in TMZ only patients who did not receive RT (HR $3.33,95 \% \mathrm{CI}, 1.31-8.45, \mathrm{P}=0.011$ ), and there was a strong trend toward a statistically significant difference between IDH mutated and wildtype groups (HR 0.35, 95\% CI, 0.12-1.06, $\mathrm{P}=0.052$ ). Overall survival, however, did differ significantly in IDH-mutated versus wildtype patients (HR 0.07, 95\% CI, 0.01-0.31, P <0.01), but final overall survival landmarks for RT-treated $v s$. TMZ-only patients have not matured. Given that the 2016 WHO guidelines requires presence of both $1 \mathrm{p} / 19 \mathrm{q}$ codeletion and IDH1/IDH2 mutation to diagnose oligodendroglioma, it is possible that the $3 \mathrm{IDH}-\mathrm{wt}$ individuals enrolled in CODEL whose tumors did not undergo sequencing harbored non-canonical IDH mutations and the 2 IDH-wt tumor may represent a rare variant of oligodendroglioma. The redesigned CODEL study comparing efficacy of chemoradiation with TMZ $v s$. PCV regimen will not have results for several more years, but is expected to be a significant advance over prior studies of oligodendroglioma by inclusion of molecularlyhomogeneous population and heralds the new standard for neuro-oncology clinical trials.

\section{Impact of molecular classification on future clinical trial design}

The 2016 update to the WHO guidelines has codified the importance of molecular classification in further categorizing distinct tumor types which are otherwise histologically similar. This reclassification has provided an important framework for how we conceptualize infiltrating gliomas (36). As further advances in glioma classification and nomenclature are made, this forces clinicians to restructure how they evaluate GBM and subsequent approaches to treatment. Given that the next interaction of WHO CNS tumor guidelines will distinguish between histopathological GBM based upon IDH1/IDH2 mutation status (as well as between classical IDH wt GBM and other IDH wt high grade gliomas, for example with $\mathrm{H} 3-\mathrm{K} 27 \mathrm{M}$ or H3G34 alterations), IDH-mutant astrocytoma WHO grade 4 
will most certainly be excluded from clinical trials geared toward GBM. However, the converse situation, whether IDH-mutant astrocytoma WHO grade 4 will henceforth be included in clinical trials of IDH-mutant astrocytoma WHO grade 2 or 3 is less clear. New glioma clinical trials will need to be designed with these distinctions in mind. Future trials may be best served by limiting enrollment to a specific molecular type of tumor. In early phase studies of safety and dosing, it could be reasonable to include all subtypes. In order to determine the early potential benefit of a treatment, it is important to first understand the safety and efficacy of the treatment modality. This comes with the understanding that the overall benefit may be minimized based on the broad application.

After determining efficacy and safety, future trials should be analyzed based on molecular classifications of CNS tumors. Thus, prior to enrollment in trials, sufficient molecular testing should be performed to render a specific diagnosis without ambiguity. In doing so, there is power to elucidate efficacy in one molecular subtype over another. Ultimately, this contemporary molecular classification paradigm necessitates that therapeutic trials focus on treatment effects as seen in individual molecular defined tumor entities. In this way, therapeutic advances can be assessed on their benefit (or lack thereof) unique to each tumor subclass. Such studies and analyses would lead to a more individualized approached to treatment to GBM, which would in turn give rise to improved survival.

\section{Conclusions}

The future of treatment for infiltrating glioma lies in an approach tailored to the potential weaknesses of each individual tumor type. While the diagnostic landscape has changed since the publication of pivotal trials, it is still important to build upon the advances studies that paved the way for treatment of GBM and other infiltrating gliomas and how their results can be interpreted under the updated classification model. Ultimately, these trials do still hold validity even in the modern era. It is essential to utilize information learned in these trials to continue evolving the approach to GBM treatment. Furthermore, it is necessary to use this new understanding when assessing ongoing clinical trials and designing future trials in order to further the understanding of treatment options for GBM. The framework provided in this paper highlights the importance of molecular markers in the treatment of GBM and aims to serve as an aid for the development of future clinical trials.

\section{Acknowledgments}

Funding: None.

\section{Footnote}

Provenance and Peer Review: This article was commissioned by the editorial office, Chinese Clinical Oncology for the series "The Evolving Landscape of the Management of Glioblastoma". The article has undergone external peer review.

Conflicts of Interest: All authors have completed the ICMJE uniform disclosure form (available at http://dx.doi. org/10.21037/cco-21-33). The series "The Evolving Landscape of the Management of Glioblastoma" was commissioned by the editorial office without any funding or sponsorship. RVL served as the unpaid Guest Editor of the series, and serves as an unpaid editorial board member of Chinese Clinical Oncology from Aug 2019 to Jul 2021. RAB reports consulting fees from IQVIA Health Technology and pariticipation in safety monitoring/advisory board for Istari Pharmaceuticals. RVL reports grants from NIH P50CA221747 and Brain Up 2137, payment/honoraria for American Physican Institute Board Review CME, Novocure speakers bureau, EBSCO publishing medical editing, MedLink Neurology medical editing, and Neurodiem presentation, as well as expert review of medical records for Loyola Medical Center, participation on advisory board for Novocure and SDP, and drug support for IIT with BMS. RS reports receiving consulting fees from CarThera, Celuarity, CranioVation, TriAct, GT Medical Technologies, and Insightec, and payment for lesions/presentations with Zailabs, as well as support for attending meetings from Novocure and receipt of materials from CarThera. The authors have no other conflicts of interest to declare.

Ethical Statement: The authors are accountable for all aspects of the work in ensuring that questions related to the accuracy or integrity of any part of the work are appropriately investigated and resolved.

Open Access Statement: This is an Open Access article distributed in accordance with the Creative Commons Attribution-NonCommercial-NoDerivs 4.0 International License (CC BY-NC-ND 4.0), which permits the noncommercial replication and distribution of the article with the strict proviso that no changes or edits are made and the 
original work is properly cited (including links to both the formal publication through the relevant DOI and the license). See: https://creativecommons.org/licenses/by-nc-nd/4.0/.

\section{References}

1. Louis DN, Perry A, Reifenberger G, et al. The 2016 World Health Organization classification of tumors of the central nervous system: a summary. Acta Neuropathol 2016;131:803-20.

2. Lukas RV, Mrugala MM. Pivotal therapeutic trials for infiltrating gliomas and how they affect clinical practice. Neurooncol Pract 2017;4:209-19.

3. Kleihues P, Cavenee WK. editors. WHO classification of tumours: pathology and genetics of tumours of the nervous system. Lyon: IARC, 2000.

4. Louis DN, Ohgaki H, Wiestler OD, et al. editors. WHO classification of tumours of the central nervous system (4th edition). Lyon: IARC, 2007.

5. Louis DN, Ohgaki H, Wiestler OD, et al. editors. WHO classification of tumours the central nervous system (revised 4th edition). Lyon: IARC, 2016.

6. Louis DN, Giannini C, Capper D, et al. cIMPACT-NOW update 2: diagnostic clarifications for diffuse midline glioma, H3 K27M-mutant and diffuse astrocytoma/ anaplastic astrocytoma, IDH-mutant. Acta Neuropathol 2018;135:639-42.

7. Brat DJ, Aldape K, Colman H, et al. cIMPACT-NOW update 3: recommended diagnostic criteria for "diffuse astrocytic glioma, IDH-wildtype, with molecular features of glioblastoma, WHO grade IV". Acta Neuropathol 2018;136:805-10.

8. Brat DJ, Aldape K, Colman H, et al. cIMPACT-NOW update 5: recommended grading criteria and terminologies for IDH-mutant astrocytomas. Acta Neuropathol 2020;139:603-8.

9. Louis DN, Wesseling P, Aldape K, et al. cIMPACTNOW update 6: new entity and diagnostic principle recommendations of the cIMPACT-Utrecht meeting on future CNS tumor classification and grading. Brain Pathol 2020;30:844-56.

10. Tesileanu CMS, Dirven L, Wijnenga, MMJ, et al. Survival of diffuse astrocytic glioma, IDH1/2 wildtype, with molecular features of glioblastoma, WHO grade IV: a confirmation of the cIMPACT-NOW criteria. Neuro Oncol 2020;22:515-23.

11. Capper D, Jones DTW, Sill M, et al. DNA methylationbased classification of central nervous system tumours.
Nature 2018;555:469-74.

12. Capper D, Stichel D, Sahm, F, et al. Practical implementation of DNA methylation and copy-numberbased CNS tumor diagnostics: the Heidelberg experience. Acta Neuropathol 2018;136:181-210.

13. Liu XY, Gerges N, Korshunov A, et al. Frequent ATRX mutations and loss of expression in adult diffuse astrocytic tumors carrying IDH1/IDH2 and TP53 mutations. Acta Neuropathol 2012;124:615-25.

14. Yan H, Parsons DW, Jin G, et al. IDH1 and IDH2 mutations in gliomas. $\mathrm{N}$ Engl J Med 2009;360:765-73.

15. Brat DJ, Verhaak RG, Aldape KD, et al. Comprehensive, integrative genomic analysis of diffuse lower-grade gliomas. N Engl J Med 2015;372:2481-98.

16. Perry A, Aldape KD, George DH, et al. Small cell astrocytoma: an aggressive variant that is clinicopathologically and genetically distinct from anaplastic oligodendrogliomas. Cancer 2004;101:2318-26.

17. Jaunmuktane Z, Capper D, Jones DTW, et al. Methylation array profiling of adult brain tumours: diagnostic outcomes in a large, single centre. Acta Neuropathol Commun 2019;7:24.

18. Meyronet D, Esteban-Mader M, Bonnet C, et al. Characteristics of H3 K27M-mutant gliomas in adults. Neuro Oncol 2017;19:1127-34.

19. Korshunov A, Capper D, Schrimpf RD, et al. Histologically distinct neuroepithelial tumors with histone 3 G34 mutation are molecularly similar and comprise a single nosologic entity. Acta Neuropathol 2016;131:137-46.

20. Sturm D, Witt H, Hovestadt V, et al. Hotspot mutations in H3F3A and IDH1 define distinct epigenetic and biological subgroups of glioblastoma. Cancer Cell 2012;22:425-37.

21. Reinhardt A, Stichel D, Schrimpf D, et al. Tumors diagnosed as cerebellar glioblastoma comprise distinct molecular entities. Acta Neuropathol Commun 2019;7:163.

22. Reinhardt A, Stichel D, Schrimpf D, et al. Anaplastic astrocytoma with piloid features, a novel molecular class of IDH wildtype glioma with recurrent MAPK pathway, CDKN2A/B and ATRX alterations. Acta Neuropathol 2018;136:273-91.

23. Korshunov A, Chavez L, Sharma T, et al. Epithelioid glioblastomas stratify into established diagnostic subsets upon integrated molecular analysis. Brain Pathol 2018;28:656-62.

24. Schreck KC, Grossman SA, Pratilas CA. BRAF Mutations and the Utility of RAF and MEK Inhibitors in Primary Brain Tumors. Cancers 2019;11:1262. 
25. Stupp R, Mason WP, van den Bent MJ, et al. Radiotherapy plus Concomitant and Adjuvant Temozolomide for Glioblastoma. N Engl J Med 2005;352:987-96.

26. Hegi ME, Diserens AC, Gorlia T, et al. MGMT gene silencing and benefit from temozolomide in glioblastoma. N Engl J Med 2005;352:997-1003.

27. Nobusawa S, Watanabe T, Kleihues P, Ohgaki H. IDH1 mutations as molecular signature and predictive factor of secondary glioblastoma. Clin Cancer Res 2009;15:6002-7.

28. Yung WK, Prados MD, Yaya-Tur R, et al. Multicenter phase II trial of temozolomide in patients with anaplastic astrocytoma or anaplastic oligoastrocytoma at first relapse. Temodal Brain Tumor Group. J Clin Oncol 1999;17:2762-71.

29. Stupp R, Taillibert S, Kanner, A, et al. Effect of TumorTreating Fields Plus Maintenance Temozolomide vs Maintenance Temozolomide Alone on Survival in Patients With Glioblastoma. JAMA 2017;318:2306-16.

30. van den Bent MJ, Baumert B, Erridge SC, et al. Interim results from the CATNON trial (EORTC study 2605322-54) of treatment with concurrent and adjuvant temozolomide for $1 \mathrm{p} / 19 \mathrm{q}$ non-co-deleted anaplastic glioma: A phase 3, randomized, open-label intergroup study. Lancet 2017;390:1645-53.

31. van den Bent MJ, Erridge S, Vogelbaum MA, et al.

Cite this article as: Singer LS, Feldman AZ, Buerki RA, Horbinski CM, Lukas RV, Stupp R. The impact of the molecular classification of glioblastoma on the interpretation of therapeutic clinical trial results. Chin Clin Oncol 2021;10(4):38. doi: $10.21037 /$ cco-21-33
PL3.3 Second interim and first molecular analysis of the EORTC randomized phase III intergroup CATNON trial on concurrent and adjuvant temozolomide in anaplastic glioma without $1 \mathrm{p} / 19 \mathrm{q}$ codeletion. Neuro Oncol 2019;21:iii3.

32. Lam K, Eldred B, Kevan B, et al. BIOM-31. Prognostic value of MGMT methylation in IDH mutant gliomas. Neuro Oncol 2020;22:ii8.

33. Bell EH, Zhang P, Fisher BJ, et al. Association of MGMT Promoter Methylation Status With Survival Outcomes in Patients With High-Risk Glioma Treated With Radiotherapy and Temozolomide: An Analysis From the NRG Oncology/RTOG 0424 Trial. JAMA Oncol 2018;4:1405-9.

34. Mathur R, Zhang Y, Grimmer MR, et al. MGMT promoter methylation level in newly diagnosed low-grade glioma is a predictor of hypermutation at recurrence. Neuro Oncol 2020;22:1580-90.

35. Jaeckle KA, Ballman KV, van den Bent M, et al. CODEL: phase III study of RT, RT + TMZ, or TMZ for newly diagnosed $1 \mathrm{p} / 19 \mathrm{q}$ codeleted oligodendroglioma. Analysis from the initial study design. Neuro Oncol 2021;23:457-67.

36. Molinaro AM, Taylor J, Wiencke JK, et al. Genetic and molecular epidemiology of adult diffuse glioma. Nat Rev Neurol 2019;15:405-17. 